\title{
An Aspect of Bilevel Indefinite Quadratic Transportation Problem Under Intuitionistic Fuzzy Environment
}

\author{
Ritu ARORA ${ }^{\mathrm{a}, 1}$, Aakanksha SINGH $^{\mathrm{b}}$ and Shalini ARORA ${ }^{\mathrm{c}}$ \\ ${ }^{a}$ Keshav Mahavidyalaya, University of Delhi, Delhi, India \\ ${ }^{\mathrm{b}}$ Research Scholar, Indira Gandhi Delhi Technical University for Women, Kashmere \\ Gate, New Delhi and Aryabhatta College, University of Delhi, Delhi, India \\ ${ }^{\mathrm{c}}$ Indira Gandhi Delhi Technical University for Women, Kashmere Gate, New Delhi, \\ India
}

\begin{abstract}
In this paper, a bilevel problem is exhibited wherein both the manufacturers and e-traders want to minimize the cost of transportation as well as deterioration cost. In todays world, reliability on online ordering has increased manifold. The "app culture" has lured the common man to prefer virtual shopping over the physical one. The consumers can order the necessities or the goods of their choice through electronic delivery apps. The manufacturers have tied up with etraders who in turn satisfy the consumers demand. With this aim, a bilevel indefinite quadratic transportation problem is dened. Sometimes it is difcult to estimate the daily demand of perishable products on a regular basis. Therefore, demand and supply parameters become uctuating in nature. To tackle this situation, bilevel indefinite quadratic transportation problem with intuitionistic fuzzy demand and supply parameters is formulated. This problem is dealt by converting fuzzy parameters into crisp ones. The transformed problem is then solved by two methods, intuitionistic fuzzy programming and fuzzy goal programming approach. To support the results, an example is also illustrated numerically representing the practical application of the problem. A comparative analysis of the solutions obtained from the two techniques is also presented. The problem is solved by computing software.
\end{abstract}

Keywords. bilevel programming, indefinite quadratic transportation, intuitionistic fuzzy programming, fuzzy goal programming, uncertainty

\section{Introduction}

Bilevel Programming Problem (BLPP) is a non-convex optimization problem. It is a decision making problem which involves the decision makers at two levels, upper level and lower level. The decision maker at lower level executes its policies after the decision maker at upper level propounds his plan of action. BLPP has numerous applications in varied practical scenarios like agriculture, banking sector, medicine, economy, energy sector as in electricity market, so on and so forth. The implementation of BLPP can also be seen in the field of transportation. Calvete et al. [1] applied ant colony optimization for production-distribution problem by formulating it as a bilevel model. Sun [2] designed the transport network problem as a bilevel model and solved it.

\footnotetext{
${ }^{1}$ Corresponding Author: Associate Professor, Keshav Mahavidyalaya, University of Delhi, Delhi, India; E-mail: ritu.arora@keshav.du.ac.in.
} 
Transportation plays an important role in any economic system bridging the gap between the consumers and the producers. Transportation models and distinct techniques for solving them have been presented by numerous authors. The simplest form of transportation problem was presented by Hitchcock [3]. Transportation problem with mixed constraints was considered by Brigden [4]. Ji and Chu [5] solved the transportation problem by the method of dualmatrix. Multi-objective transportation problem has been considered by various researchers and fuzzy programming techniques has been employed by them for solving these problems. Verma et al. [6] employed non-linear membership functions for solving multi-objective transportation problem by fuzzy programming. Ringuest and Rinks [7] proposed the algorithms for multi-objective transportation problems. Mousa [8] solved the multiobjective transportation problem by genetic algorithm and the method of TOPSIS. Kundu et al. [9] represented the multi-objective solid transportation problem in an uncertain environment and applied fuzzy programming techniques to obtain the optimal solution. Yu et al. [10] computed the compromise solution for multiobjective transportation problem where the decision makers have described the uncertainty in parameters as interval values. Shojaie and Raoofpanah [11] solved the fuzzy transportation problem by the approach of simulated annealing and genetic algorithm.

Fuzzy programming technique is a tool which facilitates the decision makers to find satisfactory solution in an optimization problem. Tanaka and Asai [12] instigated the technique of fuzzy programming. Fuzzy logic has also been used by Nittymaki and Kikuchi [13] to regulate the timings for traffic signal. In 2004, Tang et al. [14] exhibited the significance of fuzzy optimization by summarizing the models and their solution methodologies. Rommelfanger [15] described the benefits of modelling real life scenarios by fuzzy approach. Liu et al. [16] generated the optimal solution for solid transportation problem where parameters are considered as type-2 fuzzy variables. Aviso et al. [17] developed a bilevel model and applied fuzzy approach to utilize the resources and managing the waste in eco-industrial parks. The application of fuzzy approach can be seen in Ruan et al. [18] in which authors have considered the problem of medical supplies in disaster areas. Du et al. [19] solved the bilevel fuzzy programming problem for the transportation of hazardous material. Nayak and Ojha [20] solved the multi-objective non-linear programming problem by fuzzy goal programming approach. Muneeb et al. [21] formulated a fuzzy bilevel model for advertisement planning.

Although transportation problems were solved by the fuzzy programming approach, the concept of intuitionistic fuzzy sets emerged when the parameters are not precise. The perception of intuitionistic fuzzy sets was given by Atanassov [22] in 1986. Angelov [23] solved the mathematical programming problem as an application of the inuitionistic fuzzy set. In 2010, Zhang and Liu [24] applied the triangular fuzzy number for multiattitude decision making problem. Razmi et al. [25] solved the multi-objective problem by intuitionistic fuzzy approach. In 2018, Bharati and Singh [26] formulated and solved the transportation problem by intuitionistic fuzzy procedure. Ebrahimnejad and Verdegay [27] solved the transportation problem under trapezoidal intuitionistic fuzzy environment. Muthuperumal et al. [28] explained the optimization technique for unbalanced transportation problem with triangular fuzzy numbers.

Transportation problem has been considered by researchers for minimizing the cost of transportation while carrying goods from one place to another. With time it was observed that goods like fruits and vegetables may get perished or essential commodities like medicines and medical equipments such as PPE kits, oxygen cylinders etc. may get 
damaged due to poor conditions of roads or unfavourable weather conditions. The need arises to decrease the cost of damaged goods along with the cost of transportation. Thus, indefinite quadratic transportation problem (IQTP) was defined. The objective function in IQTP is a product of two linear functions, thus, minimizing the transportation cost and deterioration cost simultaneously. To the best of author's knowledge, there are not many published papers on IQTP. Bhatia [29] explained the procedure for finding the locally optimal solution for IQTP. Khurana and Arora [30] described an algorithm for finding cost-time trade off pairs for bi-criterion IQTP with fixed charge. Sokolov et al. [31] presented the solution technique for non-linear transportation problems. Table 1 represents the summary of related literature by different authors.

Table 1. Summary of related literature

\begin{tabular}{|ccc|}
\hline Author \& References & Method Used & Problem Type \\
\hline Liu \& Zhang[32] & Exact penalty method & Bilevel transportation model \\
\hline Zhang \& Gao [33] & Penalty function method & Mixed-integer non-linear BLPP \\
\hline Biswas \& Bose [34] & Fuzzy goal programming & Quadratic BLPP \\
\hline Xu \& Gang [35] & Particle swarm optimization & Fuzzy random multiobjective BLPP \\
\hline Yue et al.[36] & Fuzzy programming & Type-2 fuzzy mixed-integer BLPP \\
\hline Zhao et al.[37] & $\begin{array}{c}\text { Interactive intuitionistic } \\
\text { fuzzy method }\end{array}$ & Multilevel programming problem \\
\hline Maiti \& Roy [38] & $\begin{array}{c}\text { Intuitionistic fuzzy } \\
\text { programming }\end{array}$ & Interval and multi-choice BLPP \\
\hline Chhibber et al.[39] & $\begin{array}{c}\text { Intuitionistic fuzzy } \\
\text { programming }\end{array}$ & $\begin{array}{c}\text { Multi-objective fixed-charge solid transportation } \\
\text { problem under intuitionistic fuzzy environment }\end{array}$ \\
\hline Proposed paper & $\begin{array}{c}\text { Intuitionistic fuzzy } \\
\text { programming }\end{array}$ & $\begin{array}{c}\text { Bilevel indefinite quadratic transportation } \\
\text { problem under intuitionistic fuzzy environment }\end{array}$ \\
\hline
\end{tabular}

In the proposed problem, a bilevel model is conferred, defining IQTP at both the levels of the problem. The upper level represents the manufacturers transporting the goods like grocery, medicines, fruits, vegetables etc. to e-traders and lower level depicts the transportation from e-traders to customers in different regions of the country. The novelty of this paper is that besides minimizing the cost of transportation while carrying goods from source to destination, it also aims at minimization of the cost due to damaged goods. Moreover, due to Covid-19 pandemic, the present market scenario is fluctuating in nature. This uncertainty in supply and demand of goods is represented by intuitionistic fuzzy parameters in bilevel IQTP. The uncertain parameters are dealt using $(\alpha, \beta)$ - cut. The converted problem is then solved by intuitionistic fuzzy programming approach. The satisfactory solution for bilevel IQTP can be obtained for different values of $\alpha$ and $\beta$ provided that $\alpha+\beta \leq 1$. The paper is divided in the following sections: section 2 represents the definition of bilevel IQTP. Section 3 presents the definitions of fuzzy numbers which are vital in the elucidation of bilevel IQTP with uncertain parameters. Section 4 presents bilevel IQTP with intuitionistic fuzzy parameters and its solution methodology. Application of intuitionistic fuzzy bilevel IQTP is exhibited in section 5 followed by conclusions and prospective work in section 6 .

\section{Definition of Bilevel Indefinite Quadratic Transportation Problem}

In this section, bilevel indefinite quadratic transportation problem (BIQTP) is defined. During transportation, unpleasant weather or bad roads results in perished goods. In or- 
der to minimize the cost of damaged goods while transportation along with the transportation cost, indefinite quadratic transportation problem is considered at both the levels of (BIQTP). The objective functions at both the levels are product of two linear factors and are quasi-concave in nature. Thus, the minimum of bilevel problem will be obtained at an extreme point of the feasible region. Mathematically, (BIQTP) is defined as:

$$
(B I Q T P): \min _{Y_{1}} Z_{11}\left(Y_{1}, Y_{2}\right)=\left(e_{1}^{T} Y_{1}+e_{2}^{T} Y_{2}\right)\left(f_{1}^{T} Y_{1}+f_{2}^{T} Y_{2}\right)
$$

where $Y_{2}$ solves

$$
\left.\min _{Y_{2}} Z_{12}\left(Y_{1}, Y_{2}\right)=\left(g_{1}^{T} Y_{1}+g_{2}^{T} Y_{2}\right)\left(h_{1}^{T} Y_{1}+h_{2}^{T} Y_{2}\right) \quad \text { (for a given } Y_{1}\right)
$$

subject to

$$
\begin{gathered}
\sum_{k \in K^{\prime}} y_{j k} \leq c_{j}^{\prime} \forall j \in J^{\prime} \\
\sum_{j \in J^{\prime}} y_{j k} \geq d_{k}^{\prime} \forall k \in K^{\prime} \\
\sum_{k \in K^{\prime \prime}} y_{j k} \leq c_{j}^{\prime \prime} \forall j \in J^{\prime \prime} \\
\sum_{j \in J^{\prime \prime}} y_{j k} \geq d_{k}^{\prime \prime} \forall k \in K^{\prime \prime} \\
y_{j k} \geq 0 \forall(j, k) \in J \times K
\end{gathered}
$$

Here, $\left(e_{1}^{T} Y_{1}+e_{2}^{T} Y_{2}\right)$ represents the cost of transportation of goods from $j^{\text {th }}$ origin to $k^{\text {th }}$ destination and $\left(f_{1}^{T} Y_{1}+f_{2}^{T} Y_{2}\right)$ represents the cost of damaged goods at upper level. Similarly at the lower level, $\left(g_{1}^{T} Y_{1}+g_{2}^{T} Y_{2}\right)$ is the cost of transportation and $\left(h_{1}^{T} Y_{1}+h_{2}^{T} Y_{2}\right)$ is the cost of damaged goods.

Notations: The notations used in this mathematical model are as follows :

$\mathrm{J}, \mathrm{K}=$ Total number of sources and destinations respectively.

$J^{\prime}, K^{\prime}=$ Number of sources and destinations at upper level respectively.

$J^{\prime \prime}, K^{\prime \prime}=$ Number of sources and destinations at lower levelrespectively.

$e_{1}=\left[e_{j k}^{\prime}\right] ; f_{1}=\left[f_{j k}^{\prime}\right] ; g_{1}=\left[g_{j k}^{\prime}\right] ; h_{1}=\left[h_{j k}^{\prime}\right] \forall j \in J^{\prime}, k \in K^{\prime}$.

$e_{2}=\left[e_{j k}^{\prime \prime}\right] ; f_{2}=\left[f_{j k}^{\prime \prime}\right] ; g_{2}=\left[g_{j k}^{\prime \prime}\right] ; h_{2}=\left[h_{j k}^{\prime \prime}\right] \forall j \in J^{\prime \prime}, k \in K^{\prime \prime}$.

$J=J^{\prime} \cup J^{\prime \prime} ; J^{\prime}=\left\{1,2, \ldots, r_{1}\right\} ; J^{\prime \prime}=\left\{r_{1}+1, \ldots, r\right\}$.

$K=K^{\prime} \cup K^{\prime \prime} ; K^{\prime}=\left\{1,2, \ldots, s_{1}\right\} ; K^{\prime \prime}=\left\{s_{1}+1, \ldots, s\right\}$.

$e_{j k}^{\prime}>0 ; f_{j k}^{\prime}>0 ; g_{j k}^{\prime}>0 ; h_{j k}^{\prime}>0$ are the cost parameters for the upper level problem.

$e_{j k}^{\prime \prime}>0 ; f_{j k}^{\prime \prime}>0 ; g_{j k}^{\prime \prime}>0 ; h_{j k}^{\prime \prime}>0$ are the cost parameters for the lower level problem.

$Y_{1}=\left[y_{j k}\right], y_{j k} \geq 0 \forall j \in J^{\prime}, k \in K^{\prime}$ is the quantity transported from $j^{\text {th }}$ origin to $k^{\text {th }}$ destination at the upper level.

$Y_{2}=\left[y_{j k}\right], y_{j k} \geq 0 \forall j \in J^{\prime \prime}, k \in K^{\prime \prime}$ is the quantity transported from $j^{\text {th }}$ origin to $k^{\text {th }}$ destination at the lower level.

$c_{j}^{\prime}>0, d_{k}^{\prime}>0 \forall j \in J^{\prime}, k \in K^{\prime}$ are the supply and demand parameters at the upper level.

$c_{j}^{\prime \prime}>0, d_{k}^{\prime \prime}>0 \forall j \in J^{\prime \prime}, k \in K^{\prime \prime}$ are the supply and demand parameters at the lower level.

Feasibility conditions :

$$
\sum_{j \in J} c_{j} \geq \sum_{k \in K} d_{k} ; \sum_{j \in J^{\prime}} c_{j}^{\prime} \geq \sum_{k \in K^{\prime}} d_{k}^{\prime} ; \sum_{j \in J^{\prime \prime}} c_{j}^{\prime \prime} \geq \sum_{k \in K^{\prime \prime}} d_{k}^{\prime \prime} .
$$




\section{Basic Definitions}

Definition-1[22]: Let the universe of discourse be represented by $Z$. An intuitionistic fuzzy set in $Z$ is given by the set of three elements defined as follows:

$$
\tilde{B}^{I}=\left\{<z, \mu_{\tilde{B}^{I}}(z), \gamma_{\tilde{B}^{I}}(z)>: z \in Z\right\},
$$

where $\mu_{\tilde{B}^{I}}(z): Z \rightarrow[0,1] \quad \& \quad \gamma_{\tilde{B}^{I}}(z): Z \rightarrow[0,1]$ are functions such that $0 \leq \mu_{\tilde{B}^{I}}(z)+$ $\gamma_{\tilde{B}^{I}}(z) \leq 1 \forall z \in Z$. For each $\mathrm{z}, \mu_{\tilde{B}^{I}}(z)$ and $\gamma_{\tilde{B}^{I}}(z)$ indicates the degree of membership and degree of non-membership respectively.

Also, the degree of uncertainty of the element $z$ in the set $\tilde{B}^{I}$ is denoted by the function $\pi_{\tilde{B}^{I}}(z)$ and is defined as $\pi_{\tilde{B}^{I}}(z)=1-\left(\mu_{\tilde{B}^{I}}(z)+\gamma_{\tilde{B}^{I}}(z)\right)$. If $\pi_{\tilde{B}^{I}}(z)=0 \forall z \in Z$, then the intuitionistic fuzzy set reduces to a fuzzy set.

Definition-2[26]: Triangular Intuitionistic Fuzzy Number (TIFN)

An intuitionistic fuzzy number (IFN) of the form $\tilde{B}^{I}=\left(b_{1}, b_{2}, b_{3}\right),\left(\overline{b_{1}}, b_{2}, \overline{b_{3}}\right)$ where $\overline{b_{1}} \leq b_{1} \leq b_{2} \leq b_{3} \leq \overline{b_{3}}$ is said to be a TIFN if its membership and non-membership functions, $\mu_{\tilde{B}^{I}}(z)$ and $\gamma_{\tilde{B}^{I}}(z)$ respectively are defined as follows :

$$
\mu_{\tilde{B}^{I}}(z)=\left\{\begin{array}{ll}
\frac{z-b_{1}}{b_{2}-b_{1}} & b_{1} \leq z \leq b_{2} \\
\overline{b_{3}-z} & b_{2} \leq z \leq b_{3} \\
0 & \text { Otherwise }
\end{array} \quad \& \quad \gamma_{\tilde{B}^{I}}(z)= \begin{cases}\frac{b_{2}-z}{b_{2}-\overline{b_{1}}} & \overline{b_{1}} \leq z \leq b_{2} \\
\overline{z-b_{2}} & b_{2} \leq z \leq \overline{b_{3}} \\
1 & \text { Otherwise }\end{cases}\right.
$$

Definition-3[22]: $(\alpha, \beta)$ - cut of a triangular intuitionistic fuzzy number $\tilde{B}^{I}=\left(b_{1}, b_{2}, b_{3}\right)$, $\left(\overline{b_{1}}, b_{2}, \overline{b_{3}}\right)$ is the set of all $z$ whose degree of membership is greater than or equal to $\alpha$ and degree of non-membership is less than or equal to $\beta$. It is defined as : $\tilde{B}^{I}(\alpha, \beta)=$ $\left\{z: \mu_{\tilde{B}^{I}}(z) \geq \alpha ; \gamma_{\tilde{B}^{I}}(z) \leq \beta ; \alpha+\beta \leq 1, z \in Z\right\}$. The membership and non-membership functions are defined as $\mu_{\tilde{B}^{I}}(z): Z \rightarrow[0,1]$ and $\gamma_{\tilde{B}^{I}}(z): Z \rightarrow[0,1]$. These functions are defined such that $0 \leq \mu_{\tilde{B}^{I}}(z)+\gamma_{\tilde{B}^{I}}(z) \leq 1$. Therefore, $\alpha+\beta \leq 1$.

\section{Bilevel IQTP with Intuitionistic Fuzzy Parameters}

In fluctuating marketing scenario and due to prevailing Covid-19 pandemic conditions, the demand and the supply of goods in markets are uncertain in nature. Therefore, to address fluctuating market situations, intuitionistic fuzzy bilevel indefinite quadratic transportation problem (IFBIQTP) is defined. In this problem, uncertainty in demand and supply parameters are represented as intuitionistic fuzzy numbers.

Mathematically, intuitionistic fuzzy bilevel indefinite quadratic transportation problem with fuzzy supply and demand parameters is denoted as (IFBIQTP) and is represented as follows:

$$
(I F B I Q T P): \min _{Y_{1}} Z_{11}\left(Y_{1}, Y_{2}\right)=\left(e_{1}^{T} Y_{1}+e_{2}^{T} Y_{2}\right)\left(f_{1}^{T} Y_{1}+f_{2}^{T} Y_{2}\right)
$$

where $Y_{2}$ solves

$$
\left.\min _{Y_{2}} Z_{12}\left(Y_{1}, Y_{2}\right)=\left(g_{1}^{T} Y_{1}+g_{2}^{T} Y_{2}\right)\left(h_{1}^{T} Y_{1}+h_{2}^{T} Y_{2}\right) \quad \text { (for a given } Y_{1}\right)
$$

subject to

$$
\begin{aligned}
& \sum_{k \in K^{\prime}} y_{j k} \leq \tilde{c}_{j}^{\prime} \forall j \in J^{\prime} \\
& \sum_{j \in J^{\prime}} y_{j k} \geq \tilde{d}_{k}^{\prime I} \forall k \in K^{\prime}
\end{aligned}
$$




$$
\begin{gathered}
\sum_{k \in K^{\prime \prime}} y_{j k} \leq \tilde{c}_{j}^{\prime \prime I} \forall j \in J^{\prime \prime} \\
\sum_{j \in J^{\prime \prime}} y_{j k} \geq \tilde{d}_{k}^{\prime \prime I} \quad \forall k \in K^{\prime \prime}
\end{gathered}
$$

Also, $y_{j k} \geq 0 \forall(j, k) \in J \times K$. In this defined problem (IFBIQTP), the supply and the demand parameters are uncertain and therefore, they are represented as intuitionistic fuzzy numbers (IFN).

The membership and the non-membership functions for the supply parameters at the upper level (see Fig 1) are defined as follows with $t_{j 1}$ as the range of acceptance for the non-membership function of $\tilde{c}_{j}^{\prime}[26]$ :

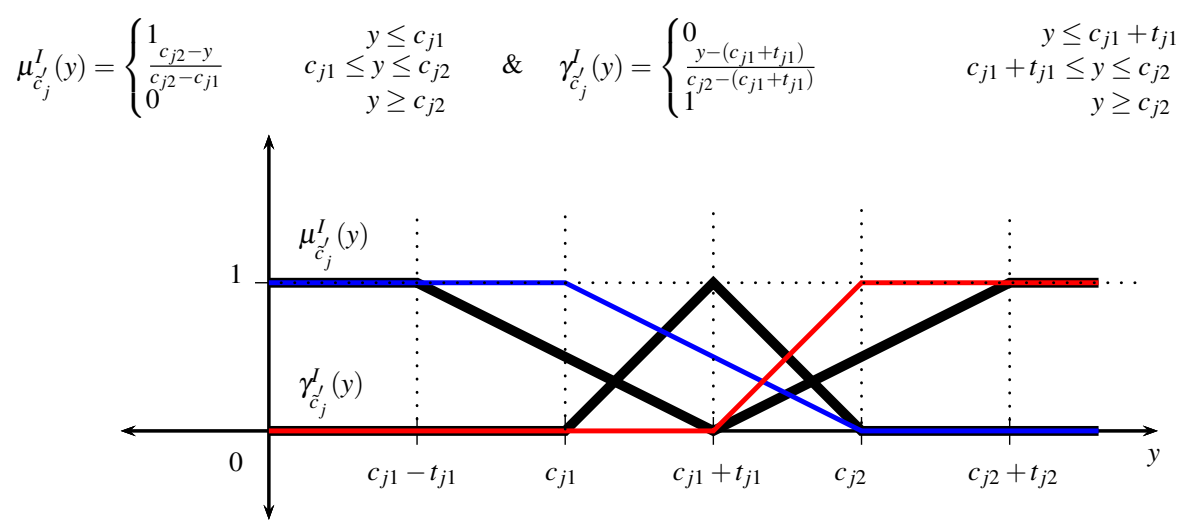

Figure 1

Again, the membership and non-membership functions for the demand parameters at the upper level (see Fig 2) are defined as follows with $t_{k 1}$ is the range of acceptance for the non-membership function of $\tilde{d}_{k}^{I}[26]$ :

$$
\mu_{\tilde{d}_{k}^{\prime}}^{I}(y)=\left\{\begin{array}{lr}
0 & y \leq d_{k 1} \\
\frac{y-d_{k 1}}{d_{k 2}-d_{k 1}} & d_{k 1} \leq y \leq d_{k 2} \\
1 & y \geq d_{k 2}
\end{array} \quad \& \quad \gamma_{\tilde{d}_{k}^{\prime}}^{I}(y)=\left\{\begin{array}{lr}
1 & y \leq d_{k 1} \\
\frac{\left(d_{k 2}-t_{k 1}\right)-y}{\left(d_{k 2}-t_{k 1}\right)-d_{k 1}} & d_{k 1} \leq y \leq d_{k 2}-t_{k 1} \\
0 & y \geq d_{k 2}-t_{k 1}
\end{array}\right.\right.
$$

Similarly, the membership function for the supply and demand parameters at the lower level can also be defined.

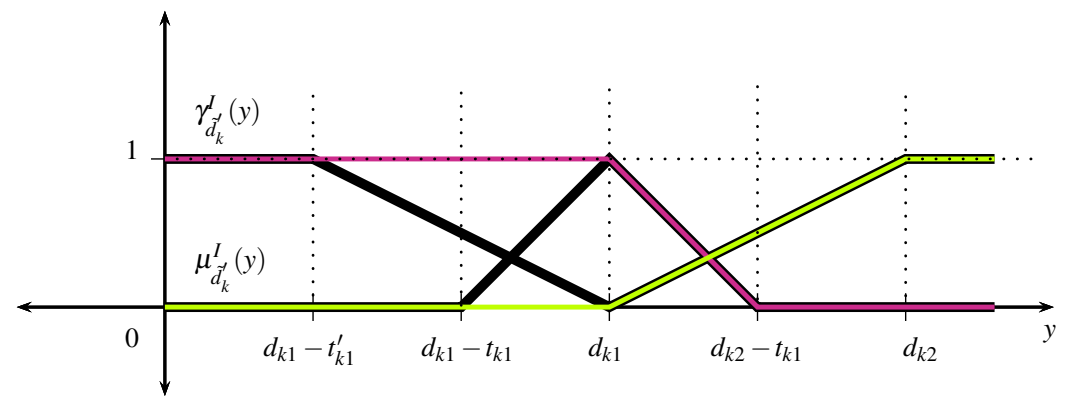

Figure 2 


\subsection{Solution Methodology}

To solve (IFBIQTP), apply $(\alpha, \beta)$ - cut to the intuitionistic fuzzy supply and demand parameters at both the levels. This will transform the set of fuzzy parameters into crisp set of elements. Applying $\operatorname{Def}(3)$ to fuzzy supply parameter at upper level we have $\mu_{\tilde{c}_{j}^{\prime}}^{I}(y) \geq$ $\alpha$ and $\gamma_{\tilde{c}_{j}^{\prime}}^{I}(y) \leq \beta$. We will get $y \leq c_{j 2}-\alpha\left(c_{j 2}-c_{j 1}\right)$ and $y \leq\left(c_{j 1}+t_{j 1}\right)+\beta\left(c_{j 2}-c_{j 1}-\right.$ $\left.t_{j 1}\right)$. Thus, the supply constraints at the upper level becomes

$$
\sum_{k \in K^{\prime}} y_{j k} \leq C_{j}^{1} \forall j \in J^{\prime}
$$

Here, $C_{j}^{1}=\operatorname{Min}\left\{c_{j 2}-\alpha\left(c_{j 2}-c_{j 1}\right),\left(c_{j 1}+t_{j 1}\right)+\beta\left(c_{j 2}-c_{j 1}-t_{j 1}\right)\right\}$. Similarly, demand constraints at the upper level problem is given by

$$
\sum_{j \in J^{\prime}} y_{j k} \geq D_{k}^{1} \forall k \in K^{\prime}
$$

Here, $D_{k}^{1}=\operatorname{Max}\left\{d_{k 1}+\alpha\left(d_{k 2}-d_{k 1}\right),\left(d_{k 2}-t_{k 1}\right)-\beta\left(d_{k 2}-t_{k 1}-d_{k 1}\right)\right\}$. Likewise, $(\alpha, \beta)-$ cut can be applied to the intuitionistic fuzzy supply and demand parameters at the lower level which will transform them into crisp sets. The problem (IFBIQTP) gets converted into the problem crisp bilevel indefinite quadratic transportation problem (CBIQTP) where supply and demand parameters at both the levels are crisp sets. (CBIQTP) is defined as follows:

$$
(C B I Q T P): \min _{Y_{1}} Z_{11}\left(Y_{1}, Y_{2}\right)=\left(e_{1}^{T} Y_{1}+e_{2}^{T} Y_{2}\right)\left(f_{1}^{T} Y_{1}+f_{2}^{T} Y_{2}\right)
$$

where $Y_{2}$ solves

$$
\left.\min _{Y_{2}} Z_{12}\left(Y_{1}, Y_{2}\right)=\left(g_{1}^{T} Y_{1}+g_{2}^{T} Y_{2}\right)\left(h_{1}^{T} Y_{1}+h_{2}^{T} Y_{2}\right) \quad \text { (for a given } Y_{1}\right)
$$

subject to

$$
\begin{aligned}
& \sum_{k \in K^{\prime}} y_{j k} \leq C_{j}^{1} \forall j \in J^{\prime} \\
& \sum_{j \in J^{\prime}} y_{j k} \geq D_{k}^{1} \forall k \in K^{\prime} \\
& \sum_{k \in K^{\prime \prime}} y_{j k} \leq C_{j}^{2} \quad \forall j \in J^{\prime \prime} \\
& \sum_{j \in J^{\prime \prime}} y_{j k} \geq D_{k}^{2} \quad \forall k \in K^{\prime \prime}
\end{aligned}
$$

Also, $y_{j k} \geq 0 \forall(j, k) \in J \times K$. From Eq.(10) and Eq.(11), we get $\sum_{k \in K^{\prime}} D_{k}^{1} \leq$ $\sum_{j \in J^{\prime}} \sum_{k \in K^{\prime}} y_{j k} \leq \sum_{j \in J^{\prime}} C_{j}^{1}$.

Thus, we get $\sum_{j \in J^{\prime}} C_{j}^{1} \geq \sum_{k \in K^{\prime}} D_{k}^{1}$. Similarly, we can have $\sum_{j \in J^{\prime \prime}} C_{j}^{2} \geq \sum_{k \in K^{\prime \prime}} D_{k}^{2}$. Since the feasibility condition at the upper level and lower level problem is described, problem (CBIQTP) is a well defined problem. 


\subsection{Intuitionistic Fuzzy Programming Approach}

We will solve the problem (CBIQTP) by Intuitionistic Fuzzy Programming Approach (IFPA). Firstly, find the maximum and minimum values of the objective functions at both the levels of (CBIQTP) with respect to the constraint set (12-15). The membership and nonmembership functions for the objective functions at both the levels are described as follows: (for $\mathrm{i}=1,2$ )

$\mu\left(Z_{1 i}\right)=\left\{\begin{array}{lr}1 & Z_{1 i} \leq Z_{1 i}^{M i n} \\ \frac{Z_{1 i}^{M a x}-Z_{1 i}}{Z_{1 i}^{M a x}-Z_{1 i}^{M i n}} & Z_{1 i}^{M i n} \leq Z_{1 i} \leq Z_{1 i}^{M a x} \\ 0 & Z_{1 i} \geq Z_{1 i}^{M a x}\end{array} \quad \& \quad \gamma\left(Z_{1 i}\right)=\left\{\begin{array}{lr}0 & Z_{1 i} \leq Z_{1 i}^{M i n} \\ \frac{Z_{1 i}-Z_{1 i}^{M i n}}{Z_{1 i}^{M a x}-Z_{1 i}^{M i n}} & Z_{1 i}^{M i n} \leq Z_{1 i} \leq Z_{1 i}^{M a x} \\ 1 & Z_{1 i} \geq Z_{1 i}^{M a x}\end{array}\right.\right.$

Let the minimum degree of satisfaction for the membership and non-membership function for the upper level problem be $\eta_{11}$ and $\eta_{11}^{\prime}$ respectively. Let $\eta_{12}$ and $\eta_{12}^{\prime}$ be the degree of satisfaction for the membership and non-membership function for the lower level problem respectively.

Let $\delta_{1}=\operatorname{Max}\left(\eta_{11}, \eta_{12}\right)$ and $\delta_{2}=\operatorname{Min}\left(\eta_{11}^{\prime}, \eta_{12}^{\prime}\right)$. The intuitionistic fuzzy programming problem for (CBIQTP) is denoted as (IFTP) and it is represented as follows :

$$
(\text { IFPP }): \operatorname{Max}\left(\delta_{1}-\delta_{2}\right)
$$

subject to

$$
\begin{array}{r}
\mu\left(Z_{11}\right) \geq \delta_{1}, \quad \mu\left(Z_{12}\right) \geq \delta_{1} \\
\gamma\left(Z_{11}\right) \leq \delta_{2}, \quad \gamma\left(Z_{12}\right) \leq \delta_{2} \\
\sum_{k \in K^{\prime}} y_{j k} \leq C_{j}^{1} \forall j \in J^{\prime} \\
\sum_{j \in J^{\prime}} y_{j k} \geq D_{k}^{1} \forall k \in K^{\prime} \\
\sum_{k \in K^{\prime \prime}} y_{j k} \leq C_{j}^{2} \forall j \in J^{\prime \prime} \\
\sum_{j \in J^{\prime \prime}} y_{j k} \geq D_{k}^{2} \forall k \in K^{\prime \prime} \\
\delta_{1} \geq \delta_{2} ; \delta_{1}+\delta_{2} \leq 1 ; \delta_{1}, \delta_{2} \in[0,1] \& y_{j k} \geq 0 \forall(j, k) \in J \times K .
\end{array}
$$

(IFPP) is solved to obtain the satisfactory solution for the problem (IFBIQTP). The computing software LINGO 17.0 is used to solve the problem (IFPP).

\subsection{Fuzzy Goal Programming Approach}

(IFBIQTP) is also solved by the fuzzy goal programming method. It is described as follows:

\section{Min $\xi$}

subject to

$$
\begin{aligned}
& Z_{11}-d_{1}^{+}+d_{1}^{-}=Z_{11}^{t} \\
& Z_{12}-d_{2}^{+}+d_{2}^{-}=Z_{12}^{t}
\end{aligned}
$$




$$
\begin{array}{r}
\sum_{k \in K^{\prime}} y_{j k} \leq C_{j}^{1} \forall j \in J^{\prime} \\
\sum_{j \in J^{\prime}} y_{j k} \geq D_{k}^{1} \forall k \in K^{\prime} \\
\sum_{k \in K^{\prime \prime}} y_{j k} \leq C_{j}^{2} \forall j \in J^{\prime \prime} \\
\sum_{j \in K^{\prime \prime}} y_{j k} \geq D_{k}^{2} \forall k \in K^{\prime \prime} \\
\xi-d_{1}^{+} \geq 0 ; \xi-d_{2}^{+} \geq 0 ; d_{1}^{+} d_{1}^{-}=0 ; d_{2}^{+} d_{2}^{-}=0 \\
0 \leq \xi \leq 1 ; y_{j k} \geq 0 \forall(j, k) \in J \times K .
\end{array}
$$

Here, $d_{1}^{+}, d_{1}^{-}, d_{2}^{+}, d_{2}^{-}$are deviational variables introduced in the objective functions. $Z_{11}^{t}, Z_{12}^{t}$ are the aspired goals of the decision makers.

\section{Application of Intuitionistic Fuzzy Bilevel IQTP}

Consider a milk manufacturing company Sukanya. The company has four plants located in Gurugram, Faridabad, Kundli and Sonipat. It transports milk and milk products like cheese, ice-cream etc. to e-traders like easy mart, milk mart, fresh mart and large basket. Due to Covid-19 pandemic, the common man place their order for their daily needs to these e-traders through electronic delivery apps. These e-traders distribute milk and milk products to customers in four different regions of Delhi; North Delhi, South Delhi, West Delhi and North-west Delhi. While delivering milk and milk product from plants to e-traders and then to customers, there is a possibility of deterioration of products like mishandling, expiry dates, temperature imbalance, melting of ice-cream and so on. The aim of the company and e-traders is to minimize the cost of transportation of products as well as the cost of deterioration. Suppose that $Z_{11}$ represents the objective function at the upper level (from plants to e-traders) and $Z_{12}$ be the objective function at the lower level (delivering products from e-traders to customers in four regions of Delhi). Table 2 and Table 3 represents the costs(in thousands) of the objective function at the upper level and at lower level respectively.

The membership functions and non-membership functions for supply and demand constraints at two levels are respectively given as follows:

$$
\begin{aligned}
& \mu_{\overline{c_{1}}}(y)=\left\{\begin{array}{lr}
1 & y \leq 31 \\
\frac{35-y}{35-31} & 31 \leq y \leq 35 \\
0 & y \geq 35
\end{array}\right. \\
& \gamma_{\overline{c_{1}}}(y)=\left\{\begin{array}{lr}
0 & y \leq 33 \\
\frac{y-33}{35-33} & 33 \leq y \leq 35 \\
1 & y \geq 35
\end{array}\right. \\
& \mu_{\overline{c_{2}}}(y)=\left\{\begin{array}{lc}
1 & y \leq 21 \\
\frac{25-y}{25-21} & 21 \leq y \leq 25 \\
0 & y \geq 25
\end{array}\right. \\
& \gamma_{c_{2}}(y)=\left\{\begin{array}{lr}
0 & y \leq 23 \\
\frac{y-23}{25-23} & 23 \leq y \leq 25 \\
1 & y \geq 25
\end{array}\right. \\
& \mu_{\overline{c_{3}}}(y)=\left\{\begin{array}{lr}
1 & y \leq 40 \\
\frac{44-y}{44-40} & 40 \leq y \leq 44 \\
0 & y \geq 44
\end{array}\right. \\
& \gamma_{\overline{c_{3}}}(y)=\left\{\begin{array}{lr}
0 & y \leq 42 \\
\frac{y-42}{44-42} & 42 \leq y \leq 44 \\
1 & y \geq 44
\end{array}\right.
\end{aligned}
$$


Table 2. Cost table for the upper level

\begin{tabular}{|c|c|c|c|c|}
\hline Plants $\quad$ E-traders & Easy mart & Milk mart & Fresh mar & Large basl \\
\hline Gurugram & 5 & 1 & 7 & $5 \rightarrow e_{j k}$ \\
\hline Faridabad & 6 & 3 & 4 & 5 \\
\hline Kundli & 5 & 1 & 4 & 2 \\
\hline Sonipat & 3 & 4 & 1 & 5 \\
\hline
\end{tabular}

Table 3. Cost table for the lower level

\begin{tabular}{|c|c|c|c|c|}
\hline $\begin{array}{ll}\text { E-traders } & \text { Regions }\end{array}$ & North Delhi & South Delhi & West Delhi & North-west Delhi \\
\hline Easy mart & 3 & 4 & 6 & $3 \rightarrow g_{j k}$ \\
\hline Milk mart & 1 & 2 & 3 & 6 \\
\hline Fresh mart & 3 & 2 & 5 & 3 \\
\hline Large basket & 1 & 6 & 4 & 5 \\
\hline
\end{tabular}

$$
\begin{aligned}
& \mu_{\overline{c_{4}}}(y)=\left\{\begin{array}{l}
1 \\
\frac{37-y}{37-31} \\
0
\end{array}\right. \\
& \begin{array}{c}
y \leq 31 \\
31 \leq y \leq 37 \\
y \geq 37
\end{array} \\
& \gamma_{\overline{c_{4}}}(y)=\left\{\begin{array}{l}
0 \\
\frac{y-32}{37-32} \\
1
\end{array}\right. \\
& 32 \leq y \leq 37 \\
& y \geq 37 \\
& \mu_{\bar{d}_{1}}(y)=\left\{\begin{array}{l}
0 \\
\frac{y-20}{24-20} \\
1
\end{array}\right. \\
& y \leq 20 \\
& 20 \leq y \leq 24 \\
& y \geq 24 \\
& \gamma_{\bar{d}_{1}}(y)=\left\{\begin{array}{l}
1 \\
\frac{22-y}{22-20} \\
0
\end{array}\right. \\
& y \leq 20 \\
& \mu_{\bar{d}_{2}}(y)=\left\{\begin{array}{l}
0 \\
\frac{y-17}{21-17} \\
1
\end{array}\right. \\
& y \leq 17 \\
& 17 \leq y \leq 21 \\
& y \geq 21 \\
& \gamma_{\overline{d_{2}}}(y)=\left\{\begin{array}{l}
1 \\
\frac{19.5-y}{19.5-17} \\
0
\end{array}\right. \\
& y \leq 17 \\
& 17 \leq y \leq 19.5 \\
& y \geq 19.5 \\
& \mu_{\bar{d}_{3}}(y)=\left\{\begin{array}{l}
0 \\
\frac{y-18}{24-18} \\
1
\end{array}\right. \\
& y \leq 18 \\
& 18 \leq y \leq 24 \\
& y \geq 24 \\
& \mu_{\bar{d}_{4}}(y)=\left\{\begin{array}{l}
0 \\
\frac{y-24}{28-24} \\
1
\end{array}\right. \\
& \begin{array}{c}
y \leq 24 \\
24 \leq y \leq 28 \\
y \geq 28
\end{array} \\
& \gamma_{\overline{d_{3}}}(y)=\left\{\begin{array}{l}
1 \\
\frac{21.5-y}{21.5-18} \\
0
\end{array}\right. \\
& y \leq 18 \\
& 18 \leq y \leq 21.5 \\
& y \geq 21.5 \\
& y \leq 24 \\
& \gamma_{\bar{d}_{4}}(y)=\left\{\begin{array}{l}
1 \\
\frac{26.5-y}{26.5-24} \\
0
\end{array}\right. \\
& 24 \leq y \leq 26.5 \\
& y \geq 26.5
\end{aligned}
$$

The intuitionistic fuzzy problem is formulated and it is solved by a computing software LINGO 17.0. Table 4 represents the satisfactory solution for the decision makers at two levels by varying the values of $\alpha$ and $\beta$. The decision makers at two levels can choose the solution best suited to them according to the demand and supply parameters. It has been 
observed from Table 4 that the values of the objective functions $Z_{11}$ and $Z_{12}$ obtained from intuitionistic fuzzy programming approach is minimum.

Table 4. Comparative Analysis

\begin{tabular}{|c|c|c|c|c|c|}
\hline \multicolumn{2}{|c|}{ Parameters } & \multicolumn{2}{c|}{$\begin{array}{c}\text { Intuitionistic Fuzzy } \\
\text { Programming Approach }\end{array}$} & \multicolumn{2}{c|}{$\begin{array}{c}\text { Fuzzy Goal } \\
\text { Programming }\end{array}$} \\
\hline$\alpha$ & $\beta$ & $Z_{11}$ & $Z_{12}$ & $Z_{11}$ & $Z_{12}$ \\
\hline 0.2 & 0.4 & 69230 & 51436 & 179703 & 185238 \\
\hline 0.2 & 0.5 & 71920 & 37300 & 186744 & 205738 \\
\hline 0.4 & 0.3 & 81939 & 51744 & 177996 & 191893 \\
\hline 0.5 & 0.2 & 71775 & 53720 & 180268 & 193154 \\
\hline 0.6 & 0.4 & 77580 & 62220 & 169212 & 173430 \\
\hline 0.8 & 0.1 & 85748 & 70132 & 168432 & 173591 \\
\hline
\end{tabular}

\section{Conclusions and Prospective Work}

Bilevel IQTP has been scrutinized in this paper when the parameters are uncertain in nature. An effort is made to exhibit the relevance of the model when e-traders are supplying milk to the consumers at their door steps. In doing so, it is required that minimum amount of milk gets deteriorated to minimize the loss. In todays world especially in this pandemic time, demand of delivery of essential commodities at door steps has upsurged exponentially. The method can also be applied to other merchandising streams involving home-delivery services. The aim of the problem is to nd a satisfactory solution for the manufacturers at upper level and e-traders at lower level. Distinct values of $\alpha$ and $\beta(\alpha+$ $\beta \leq 1$ ) will yield varied satisfactory solutions for the decision makers at two levels. The problem is solved by two approaches. One is intuitionistic fuzzy approach and another is fuzzy goal programming method. The satisfactory solution obtained by two modes is compared. It has been observed that the objective function values are minimum when the problem is solved by intuitionistic fuzzy method.

Bilevel IQTP has been considered in this paper under intuitionistic fuzzy environment. The concept of this paper can be extended to trilevel IQTP with intuitionistic fuzzy demand and supply parameters. Moreover, indeterminacy in cost coefcients can also be contemplated. Bilevel IQTP in this paper has been solved by interactive fuzzy programming technique. Authors would like to solve the problem by other methods like TOPSIS. Other algorithmic approaches like genetic algorithm, particle swarm optimization, etc. could also be examined for solving bilevel/trilevel IQTP. A similar situation with respect to time minimizing transportation problem could also be depicted.

Acknowledgements The authors are readily grateful to the reviewers for their valuable suggestions, incorporating which has helped us in improving the quality of the paper to a great extent.

\section{References}

[1] Calvete HI, Gale C, Oliveros MJ. Bilevel model for production-distribution planning solved by using ant-colony optimization. Computers \& Operations Research. 2011; 38(1): 320-327. 
[2] Sun Z. Continuous transport network design problem based on bi-level programming model. Procedia Engineering. 2016; 137: 277-282.

[3] Hitchcock, FL. The distribution of a product from several sources to numerous localities. Journal of Mathematics and Physics. 1941; 20: 224-230.

[4] Brigden MEB. A variant of the transportation problem in which the constraints are of mixed type. Journal of the Operational Research Society. 1974; 25(3): 437-445.

[5] Ji P, Chu KF. A dual-matrix approach to the transportation problem. Asia- Pacific Journal of Operational Research. 2002; 19: 35-45.

[6] Verma R, Biswal MP, Biswas A. Fuzzy programming technique to solve multi-objective transportation problems with some non-linear membership functions. Fuzzy Sets and Systems. 1997; 91(1):37-43.

[7] Ringuest JL, Rinks DB. Interactive solutions for the linear multi-objective transportation problem. European Journal of Operational Research. 1987; 32(1): 96-106.

[8] Mousa AA. Using genetic algorithms and TOPSIS technique for multi-objective transportation problem: a hybrid approach. International Journal of Computer Mathematics. 2010; 87(13): 3017-3029.

[9] Kundu P, Kar S, Maiti M. Multi-objective solid transportation problems with budget constraints in uncertain environment. International Journal of Systems Science. 2014; 45(8): 1668-1682.

[10] Yu VF, Hu KJ, Chang AY. An interactive approach for the multi-objective transportation problem with interval parameters. International Journal of Production Research. 2015; 53(4): 1051-1064.

[11] Shojaie AA, Raoofpanah H. Solving a two-objective green transportation problem by using metaheuristic methods under uncertain fuzzy approach. Journal of Intelligent \& Fuzzy Systems. 2018; 34(1): $1-10$.

[12] Tanaka H, Asai K. Fuzzy linear programming problems with fuzzy numbers. Fuzzy Sets and Systems. 1984; 13(1): 1-10.

[13] Nittymaki J, Kikuchi S. Application of fuzzy logic to the central of a pedestrian crossing signal. Transportation Research Record: Journal of the transportation Research Board. 1998; 1651(1): 30-38.

[14] Tang J, Wang D, Fung RYK, Yung KL. Understanding of fuzzy optimization: theories and methods. Journal of Systems Science and Complexity. 2004; 17(1): 117-136.

[15] Rommelfanger HJ. The advantages of fuzzy optimization in practical use. Fuzzy optimization and Decision making. 2004; 3, 295-309.

[16] Liu P, Yang L, Wang L, Li S. A solid transportation problem with type-2 fuzzy variables. Applied Soft computing. 2014; 24: 543-558.

[17] Aviso KB, Tan RR, Culaba AB, Cruz Jr. JB. Bi-level fuzzy optimization approach for water exchange in eco-industrial parks. Process Safety and Environmental Protection. 2010; 88(1): 31-40.

[18] Ruan JH, Wang XP, Chan FTS, Shi Y. Optimising the intermodal transportation of emergency medical supplies using balanced fuzzy clustering. International Journal of production Research. 2016; 54(14): 4368-4386.

[19] Du J, Li X, Yu L, Dan R, Zhou J. Multi-depot routing problem for hazardous material transportation : A fuzzy bilevel programming. Information Sciences. 2017; 399: 201-218.

[20] Nayak S, Ojha A. An approach of fuzzy and TOPSIS to bi-level multi-objective non-linear fractional programming problem. Soft Computing. 2019; 23: 5605-5618.

[21] Muneeb SM, Adhami AY, Asam Z, Jalil SA. Bi-level decision making models for advertising allocation problem under fuzzy environment. International Journal of System Assurance Engineering and Management. 2019; 10: 160-172.

[22] Atanassov KT. Intuitionistic fuzzy sets. Fuzzy Sets and Systems. 1986; 20(1): 87-96.

[23] Angelov PP. Optimization in an intuitionistic fuzzy environment. Fuzzy Sets and Systems. 1997; 66(3): 299-306.

[24] Zhang X, Liu P. Method for aggregating triangular fuzzy intuitionistic fuzzy information and its application to decision making. Technological and economic Development of economy. 2010; 16(2): 280-290.

[25] Razmi J, Jafarian E, Amin SH. An interactive fuzzy goal programming approach for finding paretooptimal solutions to multi-objective programming problems. Expert Systems with Applications. 2016; 65: 181-193.

[26] Bharati, SK, Singh, SR. Transportation problem under interval-valued intuitionistic fuzzy environment. International Journal of Fuzzy Systems.2018; 20: 15111522.

[27] Ebrahimnejad A, Verdegay, JL. A new approach for solving fully intuitionistic fuzzy transportation problems. Fuzzy optimization and decision making. 2018; 17(4): 447-474.

[28] Muthuperumal S, Titus P, Venkatachalapathy M. An algorithmic approach to solve unbalanced triangular fuzzy transportation problems. Soft Computing. 2020; 24: 18689-18698. 
[29] Bhatia HL. Indefinite quadratic solid transportation problem. Journal of Information and Optimization Sciences. 1981; 2(3): 297-303.

[30] Khurana A, Arora SR. Fixed charge bi-criterion indefinite quadratic transportation problem with enhanced flow. Investigacion Operacional. 2011; 32(2): 133-145.

[31] Sokolov AA, Tizik AP, Tsurkov VI. Iterative method for the transportation problem with additional supply and consumption points and quadratic cost. Journal of Computer and Systems Science International. 2013; 52: 588-598.

[32] Liu GS, Zhang JZ. Decision making of transportation plan, a bilevel transportation problem approach. Journal of Industrial \& Management Optimization. 2005; 1(3) : 305-314.

[33] Zhang H, Gao Z. Bilevel programming model and solution method for mixed transportation network design problem. Journal of Systems Science and Complexity. 2009; 22: 446-459.

[34] Biswas A, Bose K. A fuzzy programming approach for solving quadratic bilevel programming problems with fuzzy resource constraints. International Journal of Operational Research. 2011; 12(2): 142-156.

[35] Xu J, Gang J. Multi-objective bilevel construction material transportation scheduling in large-scale construction projects under a fuzzy random environment. Transportation Planning and Technology. 2013; 36(4): 352-376.

[36] Yue Q, Wang Y, Liu L, Niu J, Guo P, Li P. Type-2 fuzzy mixed integer bi-level programming approach for multi-source multi-user water allocation under future climate change. Journal of Hydrology. 2020; 591: 125332.

[37] Zhao X, Zheng Y and Wan Z. Interactive intuitionistic fuzzy methods for multilevel programming problems. Expert Systems with Applications. 2017; 72: 258-268.

[38] Maiti SK, Roy SK. Analysing interval and multi-choice bi-level programming for Stackelberg game using intuitionistic fuzzy programming. International Journal of Mathematics in Operational Research. 2020; 16(3): 354375.

[39] Chhibber D, Bisht DC and Srivastava PK. Pareto-optimal solution for fixed-charge solid transportation problem under intuitionistic fuzzy environment. Applied Soft Computing. 2021; 107, 107368. 